\title{
Early Esophageal Squamous Cell Carcinoma and Precancerous Lesions can be Detected by white Light Endoscopy with the Aid of White Substance
}

\section{Yingbang Wang}

Affiliated Hospital of North Sichuan Medical College

\section{Guodong Yang}

Affiliated Hospital of North Sichuan medical college

Xiaoying Zhang ( $\sim$ zxy269163@163.com )

Xiaoming Zhang

North Sichuan Medical University

\section{Linyi Wu}

North Sichuan Medical University

Xiaogang Yu

North Sichuan Medical University

Zhao Mu

North Sichuan Medical University

Yuling Chen

North Sichuan Medical University

Zhen Li

North Sichuan Medical University

\section{Research article}

Keywords: Flat esophageal mucosal lesions, Early esophageal squamous cell carcinoma, Precancerous lesions, High-grade intraepithelial neoplasia, White substance, Diagnosis

Posted Date: June 29th, 2020

DOI: https://doi.org/10.21203/rs.3.rs-37996/v1

License: (c) (1) This work is licensed under a Creative Commons Attribution 4.0 International License.

Read Full License 


\section{Early esophageal squamous cell carcinoma and precancerous lesions can be detected by white light endoscopy with the aid of white substance}

Guodong Yang, Ph.D.; Yingbang Wang, M.D.; Xiaoming Zhang, Ph.D. Xiaoying Zhang, Ph.D. " Linyi Wu, M.D. ; Xiaogang Yu, M.D.; Zhao Mu, M.D.; Yuling Chen, M.D. ; zhen Li, M.D.

\section{Declarations}

This study has been reviewed by the ethics committee

The authors have no conflict of interest to declare

Guodong Yang and Yingbang Wang contributed equally to this work. Yingbang Wang carried out the statistical analysis and drafted the manuscript. Guodong Yang participated in the study design and coordination and helped to draft the manuscript. Linyi $\mathrm{Wu}$, Xiaogang $\mathrm{Yu}$ Zhao $\mathrm{Mu}$, Yuling Chen, and zhen $\mathrm{Li}$ contributed the acquisition of clinical data. Xiaoming Zhang helped the statistical analysis. Xiaoying Zhang, manuscript critically for important intellectual content. All authors read andapproved the final manuscript.

Funding: This study was supported by the Sichuan provincial Health and Family Planning Commission Foundation (18PJ386) and Construction of Key Experimental Platform for Pancreatic Diseases in Sichuan Province (18SXHZ0371) 
Institution: ${ }^{1}$ Department of Gastroenterology and Hepatology, Affiliated Hospital of North Sichuan Medical College, Nanchong, Sichuan, China . ${ }^{2}$ Teaching, and Research Section of Parasitology, School of Basic Medicine, North Sichuan Medical College, Nanchong, Sichuan, China.

${ }^{3}$ Sichuan Key Laboratory of Medical Imaging, North Sichuan Medical College, Nanchong, Sichuan, China

${ }^{*}$ Correspondence: Xiaoying Zhang, Teaching, and Research Section of Parasitology, School of Basic Medicine, North Sichuan Medical College, Nanchong, Sichuan 637000, China (e-mail: zxy269163@163.com).

\section{Abstract}

Previous studies have shown that the presence of white substance on the esophageal mucosa is associated with tumor lesions of the flat esophageal mucosa. Since the early lesions of esophageal mucosa are mostly flat, we reasoned that the white substance might also be indicative of early esophageal squamous cell carcinoma and precancerous lesions. Objective: To investigate the diagnostic value of white substance in the detection of flat esophageal mucosal lesions and precancerous lesions associated with early esophageal squamous cell carcinoma Methods: Clinical and pathological data of patients diagnosed with flat esophageal mucosal disease were collected at the Affiliated Hospital of North Sichuan Medical College. The lesions were divided into a neoplastic group and a non-neoplastic group, and the clinicopathological differences between the 
groups were analyzed. The patients were also divided into two groups based on the presence of white substance after endoscopic examination: a "white substance" group and a "non-white substance" group. The differences in sex, age, lesion location, pathological degree, pathological nature (neoplasm and non-neoplasm), and pathological results between the two groups were analyzed. The diagnostic value of white substance for detection of early esophageal squamous cell carcinoma and related precancerous lesions was calculated and the pathological nature of white substance inferred. Results: In total, 183 patients with flat esophageal mucosal lesions were enrolled, including 92 (50.3\%) with neoplastic lesions and $91(49.7 \%)$ with non-neoplastic lesions. Forty-nine cases (26.8\%) presented white substance in the esophageal mucosa. White substance was mainly found in female patients (57.1\%), middle esophagus (75.5\%), and 60-69-year-old patients (51.0\%). Moreover, white substance was more frequently found in neoplastic than in non-neoplastic lesions (43.5\% vs. $11.0 \%, p<0.05)$. Consistently, neoplastic lesions were more represented in the white substance group than in the non-white substance group and the difference was statistically significant (79.6 vs. $20.4 \%, p<0.05)$. Fifty-one percent of the patients in the white substance group, but only $26.1 \%$ of those in the non-white substance group, had hyperkeratosis and necrosis, and the difference was statistically significant $(p<0.05)$. The diagnostic sensitivity of white 
substance for detection of early esophageal squamous cell carcinoma and precancerous lesions was $42.4 \%(95 \% C I, 32.8 \%-52.6 \%)$, the specificity $89.0 \%$ (95\% CI, 80.8\%-94.1\%), the positive predictive value $79.6 \%(95 \%$ $C I, 66.2 \%-88.7 \%)$, and the negative predictive value $60.4 \%(95 \% C I$, $53.0 \%-68.3 \%)$. Conclusions: White substance in the mucosa of flat esophageal lesions exhibited high specificity for the diagnosis of early esophageal squamous cell carcinoma and precancerous lesions. Thus, the presence of white substance in the esophageal mucosa, even in the absence of obvious lesions, may reflect the presence of latent early esophageal squamous cell carcinoma and precancerous lesions. In these cases, careful endoscopy would be recommended. White substance was associated with hyperkeratosis and necrosis. However, the mechanism behind its occurrence is still unclear and needs further investigation.

Keywords: Flat esophageal mucosal lesions; Early esophageal squamous cell carcinoma; Precancerous lesions; High-grade intraepithelial neoplasia; White substance; Diagnosis

\section{Introduction}

Esophageal cancer (EC) is the sixth deadliest cancer in the world, and is distinguished in two main pathological types: squamous cell carcinoma and adenocarcinoma ${ }^{[1,2]}$. In 2012, there were about 450000 cases of 
esophageal cancer worldwide, of which $88 \%$ were squamous cell carcinomas and $12 \%$ adenocarcinomas $^{[3]}$. Esophageal cancer has distinct regional characteristics: squamous cell carcinoma is the main type in China, the Middle East, and South Africa, while adenocarcinoma is typical of Western Europe and North America ${ }^{[4-6]}$.

Early esophageal cancer (EEC) and precancerous lesions can be treated by endoscopic submucosal dissection (ESD), and regular endoscopic follow-up can significantly reduce mortality and improve $\operatorname{prognosis}^{[4-6]}$ Some patients with early esophageal cancer have no specific clinical symptoms and poor prognosis ${ }^{[9-11]}$. In the last decade, new endoscopic techniques, such as endoscopic ultrasonography and electronic staining, have improved the detection rate of early esophageal cancer and precancerous lesions, but they are not suitable for large-scale screening ${ }^{[9-11]}$. White light endoscopy (WLE) is the most widely used endoscopic technique, and the most suitable method to screen esophageal mucosal lesions ${ }^{[13]}$. However, despite the presence of macroscopic manifestations, some esophageal squamous cell carcinomas (ESCCs) and precancerous lesions are occult and flat, and are difficult to detect by ordinary white light endoscopy ${ }^{[14]}$. A screening trial among high-risk groups of esophageal cancer showed that about $40 \%$ of patients with early esophageal squamous cell carcinoma and precancerous lesions are overlooked by white light endoscopy ${ }^{[15]}$. Although the ability of white 
light endoscopy to diagnose early esophageal cancer and precancerous lesions is limited, this approach is widely used as it is relatively easy to apply. In fact, it is still the standard method to detect esophageal lesions in countries and regions with limited healthcare resources ${ }^{[13,16]}$.

In a previous study, we found that white substance often appears in flat-type esophageal mucosal lesions, and has a high diagnostic specificity for flat esophageal mucosal neoplasia ${ }^{[17]}$. Early esophageal squamous cell carcinoma and precancerous lesions often appear in the form of flat esophageal mucosal lesions, which are, therefore, considered diagnostically relevant ${ }^{[18]}$. However, since the latter study was retrospective, the nature of white substance could not be explored. We speculated that the white substance might be associated with hyperkeratosis, inflammatory necrosis or other lesions. Thus, to investigate the diagnostic value of white substance for early esophageal squamous cell carcinoma and precancerous lesions, and explore its pathological properties, we retrospectively collected and analyzed the clinicopathological data of patients diagnosed with flat esophageal mucosal lesions at the Affiliated Hospital of North Sichuan Medical College from 2017 to 2019. The demonstration of a diagnostic value of white substance for the detection of early esophageal squamous cell carcinoma and precancerous lesions may substantially help endoscopists improve the diagnostic rate of white light endoscopy, which is 
particularly important for high-risk areas with limited healthcare resources.

\section{Methods and Patients}

\subsection{Definitions}

The following definitions were used in this study:

White substance: the white substance should meet the following characteristics: 1) present a clear boundary; 2) present a white adherent surface; 3 ) it should not be removable by washing (Fig. 1).

Flat esophageal mucosal lesions: the macroscopic morphology of esophageal mucosal lesions conformed to Paris type 0-II, with rough or uneven color change of the esophageal mucosa or iodine staining without color changes, with or without blood texture changes. The nature of the lesions could be either neoplastic or non-neoplastic (Fig. 2).

Neoplastic lesions: included early esophageal squamous cell carcinoma, low-grade intraepithelial neoplasia (LIN), and high-grade intraepithelial neoplasia (HIN).

Non-neoplastic lesions: chronic inflammation and squamous hyperplasia/thickening, in accordance with the morphological characteristics of flat esophageal mucosal lesions. 


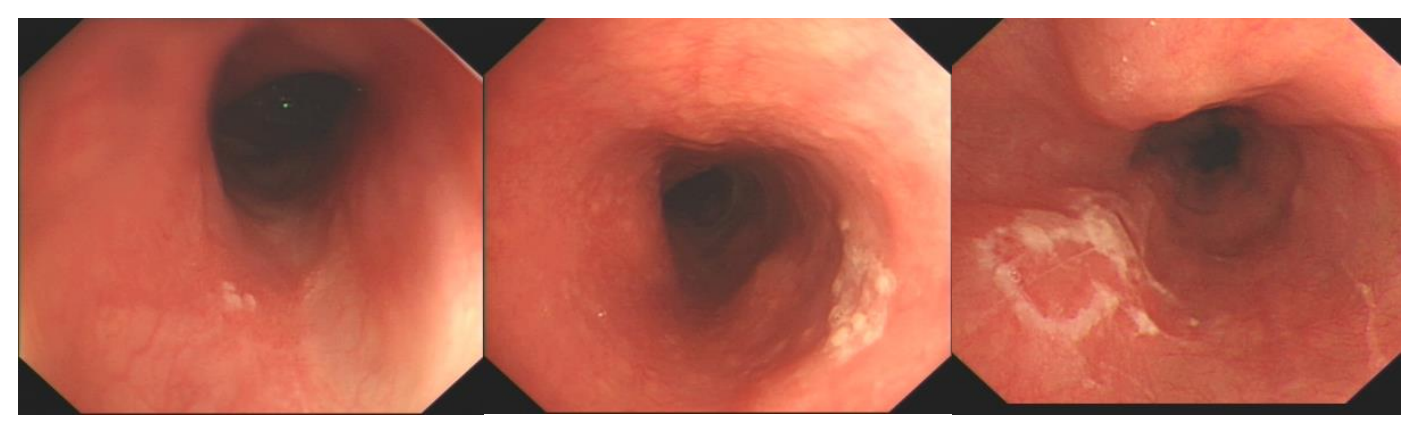

Fig. 1 White substance has a clear boundary, can be distributed in flakes or as single spots, and cannot be easily washed away with water. Its shape is different from that of esophageal leukoplakia.

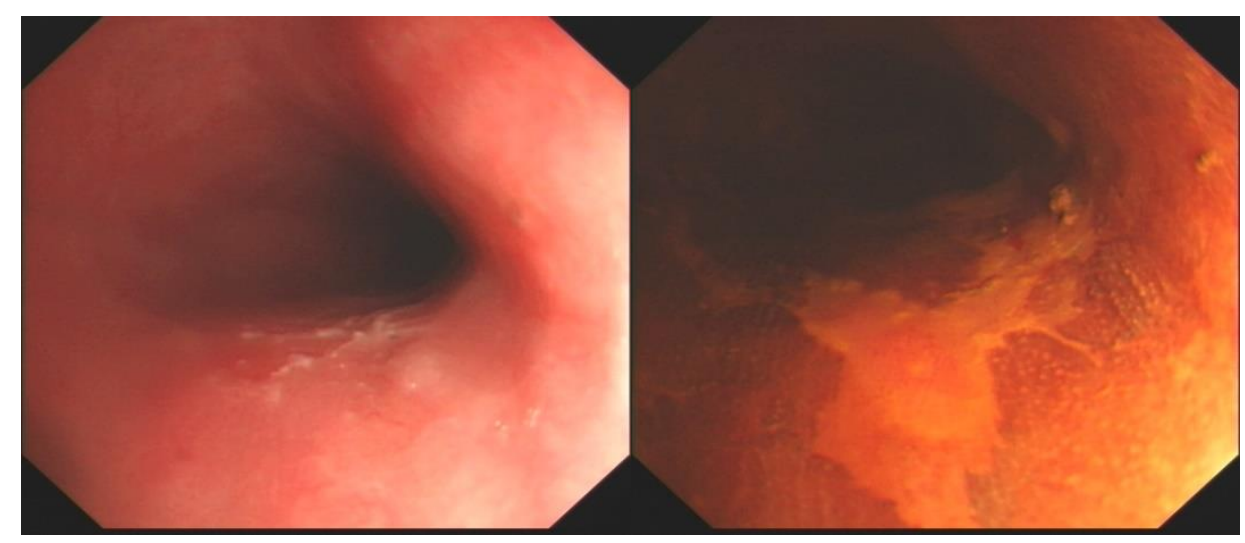

Fig. 2 By white light endoscopy, the esophageal mucosa appears slightly rough and focally eroded but there is no obvious narrowing, ulcer or mass. The focal achromatic area can be detected by iodine staining.

Hyperkeratosis: this term refers to the excessive thickening of the cuticle of mucocutaneous skin, which can occur in any part of the human body, often because of the exposure to chemicals or viruses (Fig. 3). 


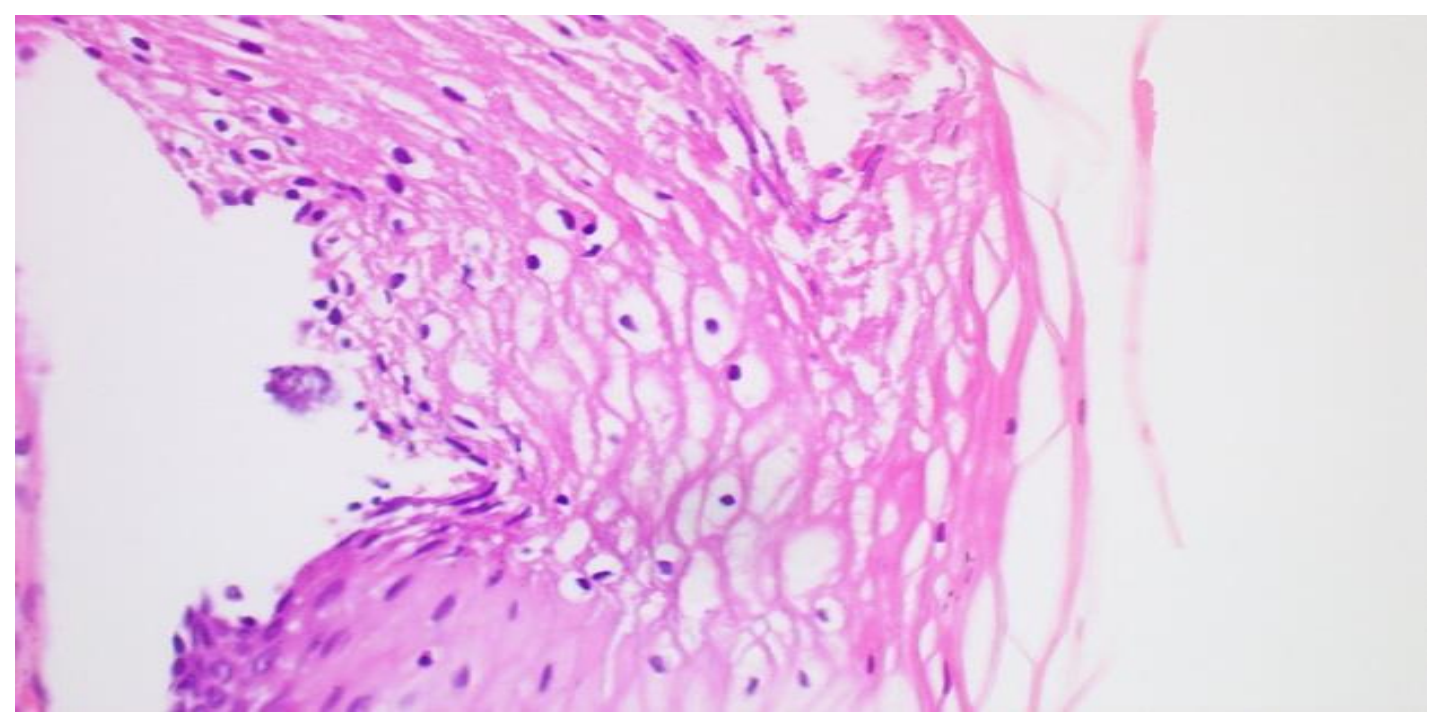

Fig. 3 Hyperkeratosis $(400 \times$ magnification): the cell boundaries are unclear, the nucleus is not observable, and hyperkeratosis is clearly accompanied by the thickening of the granular layer and by obviously abnormal hyaluronic granules.

\subsection{Patients}

The patients included in this study underwent endoscopy at the Gastroenterology Center of the Affiliated Hospital of North Sichuan Medical College from 2017 to 2019 for various reasons. They were diagnosed with esophageal mucosal lesions after examination by different endoscopists. The patients diagnosed with early esophageal squamous cell carcinoma and precancerous lesions underwent the relevant auxiliary examinations at the Affiliated Hospital of North Sichuan Medical College to exclude metastasis, followed by ESD. Complete auxiliary examination results and postoperative pathological data were available for these patients.

\subsection{Research foundation}


All endoscopic images were obtained from the Digestive Endoscopy Center of the Affiliated Hospital of North Sichuan Medical College, an advanced medical center in a Chinese area with high incidence of EC. All endoscopic examinations and diagnoses were performed by endoscopists with more than 2 years of experience. High-resolution white light imaging endoscopy (EVIS LUCERA CLV-260; Olympus, Tokyo, Japan) and a video processor (EVIS LUCERA CLV-260; Olympus) were used. The pathological specimens were evaluated by senior pathologists. The study was approved by the Ethics Committee of the Affiliated Hospital of North Sichuan Medical College, and because of its retrospective nature, informed consent from patients was not required.

\subsection{Inclusion and exclusion criteria}

2.4.1. Inclusion criteria:

(1) Esophageal lesions conformed to the characteristics of flat esophageal mucosal lesions.

(2) Complete pathological data and corresponding auxiliary examination results were available.

(3) The endoscopic picture was clear.

2.4.2. Exclusion criteria:

(1) The patients presented other neoplasias or immunodeficiency.

(2) The pathological specimens were seriously deteriorated and could not 
be examined.

(3) The pathological findings were suggestive of adenocarcinoma or Barrett's esophagus.

\subsection{Methods}

Step1: The clinicopathological data (including general information, endoscopic images, pathological results, and other auxiliary examination results) of patients with flat esophageal mucosal lesions diagnosed in our hospital from 2017 to 2019 were retrospectively collected and analyzed according to the research objectives, and all eligible patients were screened according to the inclusion and exclusion criteria.

Step2: The pathological specimens of the patients included in the study were interpreted twice by senior pathologists and the pathological results were recorded. According to the nature of the lesions, the selected patients were divided into a neoplastic lesion group and a non-neoplastic lesion group, and the clinicopathological characteristics of each group were recorded.

Step 3: The endoscopic images were reviewed by two experienced endoscopists, and the presence and distribution of white substance were recorded. The patients were divided into a 'white substance group' and a 'non-white substance group', and the clinicopathological characteristics of the two groups were recorded. 
Step 4: A comprehensive analysis of clinicopathological data in each group was performed by using professional statistical methods and software (Fig. 4).

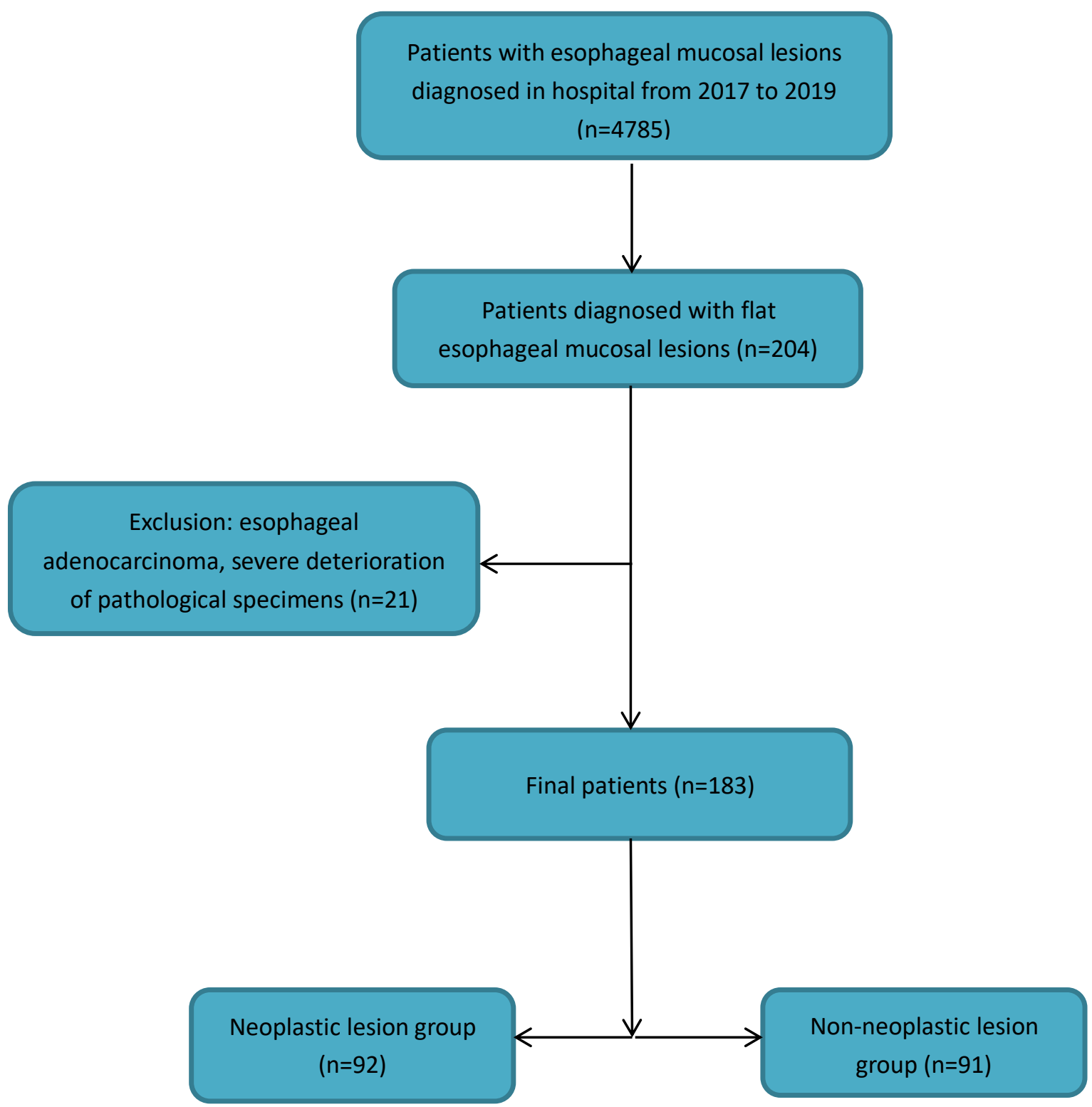

Figure 4 Flow diagram of patients enrolled in this study

\subsection{Statistical methods}


Data were statistically analyzed by using SPSS V.22.0 software, and categorical data were compared using the chi-square test or Fisher's exact test. To evaluate the diagnostic value of white substance for early esophageal squamous cell carcinoma and precancerous lesions, the sensitivity, specificity, and corresponding 95\% confidence interval (CI) were considered. The positive predictive value (PPV) and negative predictive value (NPV) were calculated using standard formulas. Two-tailed $p<0.05$ was considered statistically significant.

\section{Results}

From 2017 to 2019,4785 patients underwent upper gastrointestinal endoscopy and pathological biopsy at the Endoscopy Center of North Sichuan Medical Affiliated Hospital due to the presence of esophageal mucosal lesions. Of the 204 patients who met the inclusion criteria, 21 were excluded due to the presence of other neoplasias or severe alteration of the pathological specimens. Finally, the clinicopathological data of 183 patients with flat esophageal mucosal lesions were statistically analyzed.

\subsection{Age distribution of neoplastic and non-neoplastic patients with flat esophageal mucosal lesions}

The mean age of 183 patients with flat esophageal mucosal lesions was 61.7 years. The number of patients with neoplastic lesions increased 
with age, reaching a peak of $49(38.8 \%)$ at the age of 60-69 years, and declining to $16(17.4 \%)$ at the age of $70-79$ years. Patients with non-neoplastic lesions were rare before age 40 , but their number was comparable across age groups after age 40 (Fig. 5).

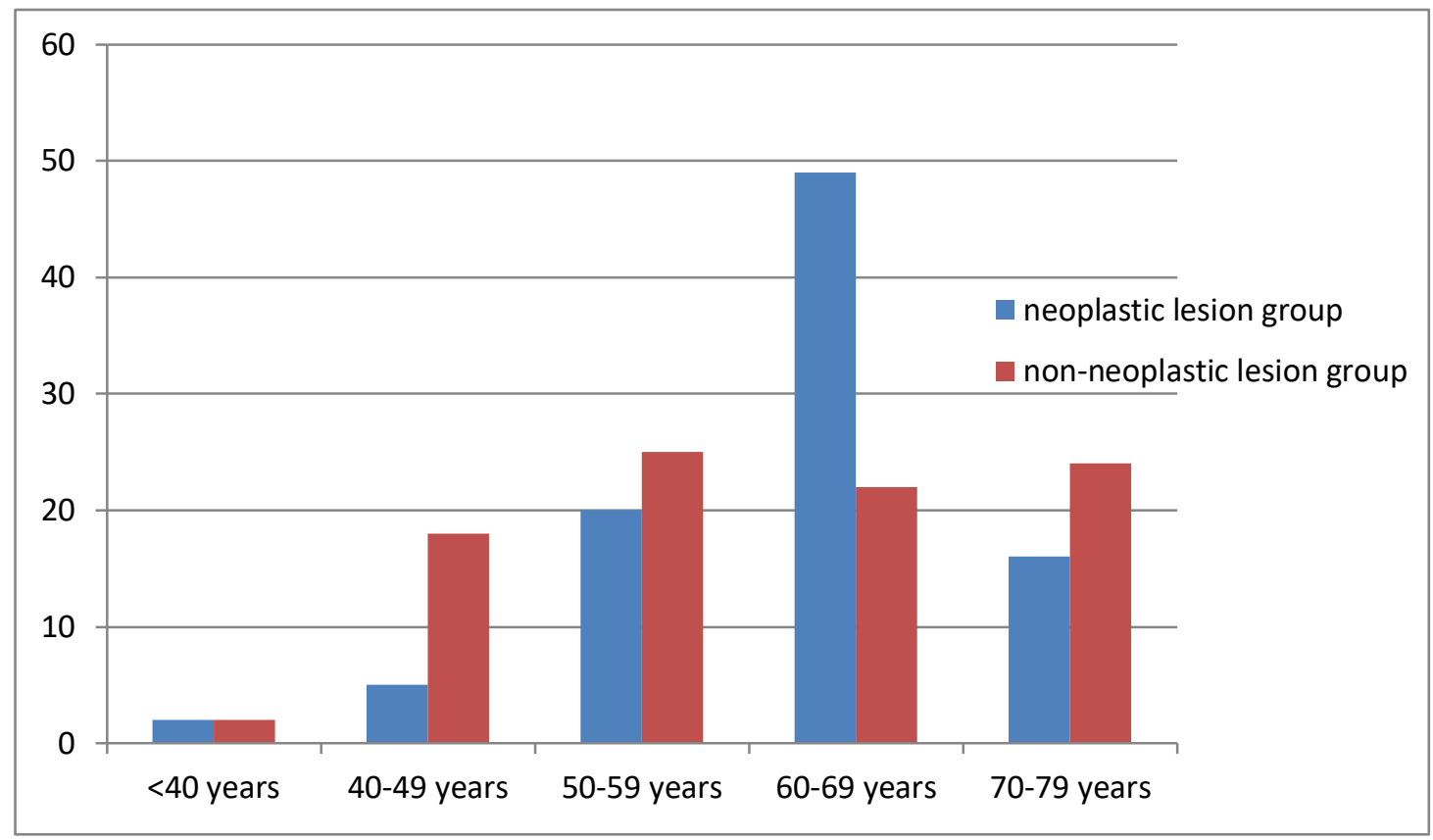

Figure 5. Age distribution of neoplastic and non-neoplastic lesions

\subsection{Clinical and pathological characteristics of patients with flat esophageal mucosal lesions}

Flat esophageal mucosal lesions were more frequent among male than female patients $(57.9 \%$ vs.42.1\%). Most lesions were located in the middle part of the esophagus (71.7\%). The most numerous age groups consisted of patients aged 60-69 years (71 patients, 38.8\%). Of these, 92 (50.3\%) had neoplastic lesions and 91 (49.7\%) non-neoplastic lesions. Pathological examination revealed 47 cases of hyperkeratosis $(25.7 \%), 8$ cases of necrosis $(4.4 \%), 60$ cases of hyperkeratosis combined with 
necrosis $(32.8 \%), 68$ cases without keratosis or necrosis $(37.2 \%)$. White substance was present in 49 patients with flat esophageal mucosal lesions, accounting for $26.8 \%$ of the total population. The basic characteristics of patients with flat esophageal mucosal lesions are shown in Table 1.

Table 1. Basic characteristics of patients with flat esophageal mucosal lesions $(\mathrm{n}=183)$

\begin{tabular}{ll}
\hline items & results \\
\hline Sex, n (\%) & \\
Males & $106(57.9 \%)$ \\
Females & $77(42.1 \%)$ \\
Age, $\mathrm{n}(\%)$ & \\
$<40$ years & $4(2.2 \%)$ \\
$40-49$ years & $23(12.6 \%)$ \\
$50-59$ years & $45(24.6 \%)$ \\
$60-69$ years & $71(38.8 \%)$ \\
$70-79$ years & $40(21.9 \%)$ \\
Lesion location, $\mathrm{n}(\%)$ & $81(4.4 \%)$ \\
Upper segment esophagus & $47(25.7 \%)$ \\
Middle segment esophagus & $22(12.0 \%)$ \\
Lower segment esophagus & $132(72.1 \%)$ \\
Nature of the lesion, $\mathrm{n}(\%)$ & $29(15.9 \%)$ \\
Neoplastic lesions & \\
Non-neoplastic lesions & \\
Hathological results, $\mathrm{n}(\%)$ & \\
Necrosis & \\
\hline
\end{tabular}


Hyperkeratosis with necrosis

No hyperkeratosis or necrosis

White substance, n (\%)

Presence

Absence
$60(32.8 \%)$

$68(37.2 \%)$

$49(26.8 \%)$

$134(73.2 \%)$

\subsection{Clinical and pathological differences between patients with}

neoplastic and non-neoplastic lesions

Neoplastic lesions were mostly found in males (53.3\%). The lesions were mostly located in the middle esophagus $(81.5 \%)$, and patients aged 60-69 years presented the largest proportion of lesions (53.3\%). Hyperkeratosis and necrosis were more common in patients with neoplastic lesions $(63.0 \%)$, while $69.2 \%$ patients with non-neoplastic lesions had neither hyperkeratosis nor necrosis. White substance was mostly found in the neoplastic lesion group (43.5\% vs.11.0\%, $p<0.001)$. The clinical and pathological characteristics of the neoplastic and non-neoplastic group are shown in Table 2.

Z

Table 2. Clinical and pathological characteristics of neoplastic and non-neoplastic patients

\begin{tabular}{llll}
\hline items & $\begin{array}{l}\text { Neoplastic group } \\
(\mathrm{n}=92)\end{array}$ & $\begin{array}{l}\text { Non-neoplastic group } \\
(\mathrm{n}=91)\end{array}$ & $p$-value \\
\hline
\end{tabular}




\begin{tabular}{|c|c|c|c|}
\hline Sex, n $(\%)$ & & & $P=0.199$ \\
\hline Males & $49(53.3 \%)$ & $57(62.6 \%)$ & \\
\hline Females & $43(46.7 \%)$ & $34(37.4 \%)$ & \\
\hline Age, $\mathrm{n}(\%)$ & & & $P<0.001$ \\
\hline$<40$ years & $2(2.2 \%)$ & $2(2.2 \%)$ & \\
\hline $40-49$ years & $5(5.4 \%)$ & $18(19.8 \%)$ & \\
\hline $50-59$ years & $20(21.7 \%)$ & $20(21.7 \%)$ & \\
\hline $60-69$ years & $49(53.3 \%)$ & $22(24.1 \%)$ & \\
\hline $70-79$ years & $16(17.4 \%)$ & $24(26.4 \%)$ & \\
\hline Lesion location, $\mathrm{n}(\%)$ & & & $P=0.016$ \\
\hline Upper esophagus & $8(8.7 \%)$ & $14(15.4 \%)$ & \\
\hline Middle esophagus & $75(81.5 \%)$ & $57(62.6 \%)$ & \\
\hline Lower esophagus & $9(9.8 \%)$ & $20(22.0 \%)$ & \\
\hline Pathological results, n (\%) & & & $p<0.001$ \\
\hline Necrosis & $2(2.2 \%)$ & $6(6.6 \%)$ & \\
\hline Hyperkeratosis & $27(29.3 \%)$ & $20(22.0 \%)$ & \\
\hline $\begin{array}{l}\text { Hyperkeratosis with } \\
\text { necrosis }\end{array}$ & $58(63.0 \%)$ & $2(2.2 \%)$ & \\
\hline $\begin{array}{l}\text { No hyperkeratosis or } \\
\text { necrosis }\end{array}$ & $5(5.4 \%)$ & $63(69.2 \%)$ & \\
\hline White substance, $\mathrm{n}(\%)$ & & & $p<0.001$ \\
\hline Presence & $39(43.5 \%)$ & $10(11.0 \%)$ & \\
\hline Absence & $53(56.5 \%)$ & $81(89.0 \%)$ & \\
\hline
\end{tabular}

\subsection{Clinicopathological differences between the white substance}




\section{group and the non-white substance group}

Of the patients included in the study, 49 had white substance in the esophageal mucosa, accounting for $26.8 \%$ of total patients. White substance was more common in female patients $(57.1 \%)$, predominantly located in the middle esophagus (78.38\%), and was most frequently found in the 60-69 year age group (51.0\%). Patients of the white substance group had more neoplastic lesions than non-neoplastic lesions (79.6\% vs 20.4\%, $p<0.05)$, whereas there was no statistical significance in the neoplastic lesion group $(p>0.05)$. The hyperkeratosis/necrosis combination was observed in $51 \%$ of the patients with white substance, but only in $26.1 \%$ of those of the non-white substance group, and the difference was statistically significant $(p<0.05)$. The differences in clinicopathological characteristics between patients with and without white substance are shown in Table 3.

Table 3 Clinicopathological differences between patients with and without white substance.

\begin{tabular}{lllc}
\hline items & Presence $(\mathbf{n}=49)$ & Absence $(\mathbf{n = 1 3 4})$ & p-value \\
\hline Sex, n (\%) & & & \\
Males & $21(42.9 \%)$ & $85(63.4 \%)$ & \\
Females & $28(57.1 \%)$ & $49(36.6 \%)$ & \\
Age, n $(\%)$ & & $p=0.013$ \\
$<40$ years & $3(6.1 \%)$ & $1(0.7 \%)$ &
\end{tabular}




\begin{tabular}{|c|c|c|c|}
\hline $40-49$ years & $5(10.2 \%)$ & $18(13.4 \%)$ & \\
\hline $50-59$ years & $11(22.4 \%)$ & $34(25.4 \%)$ & \\
\hline $60-69$ years & $25(51.0 \%)$ & $46(34.3 \%)$ & \\
\hline $70-79$ years & $5(10.2 \%)$ & $35(26.1 \%)$ & \\
\hline Lesion location, n (\%) & & & $p=0.424$ \\
\hline Upper esophagus & $7(14.3 \%)$ & $15(11.2 \%)$ & \\
\hline Middle esophagus & $37(75.5 \%)$ & $95(70.9 \%)$ & \\
\hline Lower esophagus & $5(10.2 \%)$ & $24(17.9 \%)$ & \\
\hline Nature of the lesion, $\mathrm{n}(\%)$ & & & $p<0.001$ \\
\hline Non-neoplastic lesions & $10(20.4 \%)$ & $81(60.4 \%)$ & \\
\hline Neoplastic lesions & $39(79.6 \%)$ & $53(39.6 \%)$ & $p=0.962$ \\
\hline LIN & $10(27.03 \%)$ & $15(22.73 \%)$ & \\
\hline HIN & $20(54.05 \%)$ & $25(54.55 \%)$ & \\
\hline Early carcinoma & $9(18.92 \%)$ & $13(22.73 \%)$ & \\
\hline Pathological results, n (\%) & & & $p=0.011$ \\
\hline Necrosis & $2(4.2 \%)$ & $6(4.5 \%)$ & \\
\hline Hyperkeratosis & $11(22.4 \%)$ & $36(26.9 \%)$ & \\
\hline Hyperkeratosis with necrosis & $25(51 \%)$ & $35(26.1 \%)$ & \\
\hline No hyperkeratosis or necrosis & $11(22.4 \%)$ & $57(42.5 \%)$ & \\
\hline
\end{tabular}

\subsection{Diagnostic value of white substance for early esophageal squamous cell carcinoma and precancerous lesions}

The overall distribution rate of white substance was $26.8 \%, 42.4 \%$, and $11.0 \%$ among patients with flat esophageal mucosal lesions, neoplastic lesions, and non-neoplastic lesions, respectively, and the 
differences were statistically significant $(p<0.05)$. The prevalence of early esophageal squamous cell carcinoma and precancerous lesions among patients with flat esophageal mucosal lesions was 50.3\% (95\% CI, 43.1\%-57.4\%). The diagnostic sensitivity of white substance in the esophageal mucosa for early esophageal squamous cell carcinoma and precancerous lesions was $42.4 \%$ (95\% CI, 32.8\% -52.6\%), the specificity $89.0 \%$ (95\% CI, $80.8 \%-94.1 \%$ ), the PPV $79.6 \%$ (95\% CI, $66.2 \%-$ 88.7\%), and the NPV 60.4\% (95\% CI, 53.0\% -68.3\%) (Table 4).

Table 4. Diagnostic value of white substance for early esophageal squamous cell carcinoma and intraepithelial neoplasia

\begin{tabular}{lc}
\hline items & results \\
\hline Prevalence $(95 \% \mathrm{CI})$ & $50.3 \%(43.1-57.4 \%)$ \\
Sensitivity (95\% CI) & $42.4 \%(32.8-52.6 \%)$ \\
Specificity (95\% CI) & $89.0 \%(80.8-94.1 \%)$ \\
Positive predictive value (95\% CI) & $79.6 \%(66.2-88.7 \%)$ \\
Negative predictive value (95\% CI) & $60.4 \%(53.0-68.3 \%)$ \\
\hline
\end{tabular}

\section{Discussion}

The treatment and prognosis of esophageal cancer depend on the stage of the lesion. Therefore, early diagnosis is extremely important ${ }^{[19]}$. White light endoscopy is cost-effective and can be carried out in primary medical units. It is still the main screening method in areas with high 
incidence of ESCC. Some early ESCC and precancerous lesions have occult onset, In these patients, the mucosa appears macroscopically normal. These lesions are difficult to detect by white light endoscopy, resulting in missed diagnosis ${ }^{[20]}$. In this study, white substance could be observed by white light endoscopy without additional staining or other high-end endoscopic techniques, thus minimizing the risk of missed diagnosis.

Our data showed that the incidence of early esophageal squamous cell carcinoma and precancerous lesions among patients with flat esophageal mucosal lesions was low before 40 years of age, and gradually increased after this age, reaching a peak at 60-69 years, with lesions mostly located in the middle esophagus. Therefore, in areas with high incidence of ESCC, the presence of early esophageal cancer and precancerous lesions should be monitored in patients over 40 years of age presenting with flat esophageal mucosal lesions in the middle esophagus.

The frequency of white substance was much higher among patients with neoplastic lesions compared to patients with non-neoplastic lesions (43.5\% vs.11.0\%, $p<0.05)$, and the frequency of neoplastic lesions in the white substance group was significantly higher than in the non-white substance group $(79.6 \%$ vs.39.6\%, $p<0.05)$. This indicated that white substance was closely related to early esophageal squamous cell carcinoma and precancerous lesions, and could be used as an endoscopic 
marker.

White substance exhibited high specificity and PPV for the diagnosis of early esophageal squamous cell carcinoma and precancerous lesions, independently of lesion location and extent. The detection of this white material anywhere in the esophageal mucosa by white light endoscopy should alert clinicians to the chance of early esophageal squamous cell carcinoma and precancerous lesions, even in the absence of obvious abnormalities. Notably, due to the lack of adequate sensitivity, high-quality endoscopy should be performed whenever possible in patients at high risk for esophageal neoplasia in high-risk areas, even in the absence of white substance in the esophageal mucosa.

The data of this study indicated that the presence of white substance might reflect hyperkeratosis with some necrosis. Hyperkeratosis is a common lesion in the head and neck, and its risk factors include smoking, alcohol, age, and low socioeconomic status ${ }^{[21-23]}$. There was no hyperkeratosis in normal oral, esophageal and throat mucosa. A previous study showed that hyperkeratosis is associated with esophageal squamous epithelial neoplasia and head and neck squamous epithelial lesions, but the specific pathogenetic mechanism remains unclear ${ }^{[24]}$. In this study, hyperkeratosis combined with necrosis was found to be much more frequent in patients with neoplastic lesions than in those with non-neoplastic lesions $(63.0 \%$ vs.2.2\%, $p<0.05)$, suggesting that the 
combination of hyperkeratosis and necrosis was associated with early esophageal squamous cell carcinoma and related precancerous lesions. However, to date, few studies have investigated the association between hyperkeratosis and ESCC, and the pathogenetic mechanism is still unclear.

The results of this study showed that white substance was more common in females and patients aged 60-69 years $(p<0.05)$, but its incidence was not affected by lesion location and extent (low-grade, high-grade intraepithelial neoplasia, early squamous cell carcinoma) ( $p>$ 0.05). Previous studies showed that the distribution of white substance is not affected by sex or age $(p>0.05)^{[17]}$. This discrepancy may be because these previous studies were retrospective, and based on incomplete data on invasion depth, tumor metastasis, and other aspects. This may have led to the consideration of advanced tumors or adenocarcinomas as neoplastic lesions. In the present study, distant metastasis was excluded by auxiliary examination in all patients with neoplastic lesions, which were confirmed to be early esophageal squamous cell carcinomas and precancerous lesions by postoperative pathological examination.

The white substance of esophageal mucosa is of significance in the diagnosis of early esophageal squamous cell carcinoma and precancerous lesions. Moreover, it can be observed by white light endoscopy without additional staining or other high-level endoscopic techniques. White 
substance may help endoscopists detect hidden esophageal mucosal lesions, especially in primary medical units or areas with poor medical resources, to reduce missed diagnosis and improve prognosis.

There are a few shortcomings in this study. For example, the number of patients included in the study was small, and the results could be biased.

\section{Conclusions}

Among subjects with flat esophageal mucosal lesions, white substance was most common in female patients of 60-69 years of age, and was predominantly found in the middle esophagus. White substance was a highly specific diagnostic marker for early esophageal squamous cell carcinoma and precancerous lesions. Therefore, the evaluation of white substance may support white light endoscopy in the detection of occult early esophageal squamous cell carcinoma and precancerous lesions. On the other hand, since the diagnostic sensitivity was low, the absence of white substance from flat esophageal mucosal lesions should not induce the clinicians to 'let their guard down'. Our data showed that the pathological consequence of white substance was hyperkeratosis combined with partial necrosis, albeit the specific mechanism underlying white substance occurrence is still unclear and requires further investigation. 


\section{References}

[1] Murphy G, McCormack V, Abedi-Ardekani B, et al. International cancer seminars: a focus on esophageal squamous cell carcinoma[J]. Ann Oncol, 2017,28(9):2086-2093.

[2] Alsop BR, Sharma P. Esophageal Cancer[J]. Gastroenterol Clin North Am, 2016,45(3):399-412.

[3] Arnold M, Soerjomataram I, Ferlay J, et al. Global incidence of oesophageal cancer by histological subtype in 2012[J]. Gut, 2015,64(3):381-387.

[4] He L, Jin-Hu F, You-Lin Q, et al. Epidemiology, etiology, and prevention of esophageal squamous cell carcinoma in China[J]. Cancer Biol Med, 2017,14(1):33-41.

[5] Abnet C, Arnold M, Wei W. Epidemiology of Esophageal Squamous Cell Carcinoma[J]. Gastroenterology, 2018,154(2):360-373.

[6] Steevens J, Botterweck AM, Dirx MJM, et al. Trends in incidence of oesophageal and stomach cancer subtypes in Europe[J]. Eur J Gastroenterol Hepatol, 2010,22(6):669-678.

[7] Kandiah K, Chedgy FJ, Subramaniam S, et al. Early squamous neoplasia of the esophagus: The endoscopic approach to diagnosis and management[J]. Saudi J Gastroenterol, 2017,23(2):75-81.

[8] Ning B, Abdelfatah M, Othman MO. Endoscopic submucosal dissection and endoscopic mucosal resection for early stage esophageal cancer[J]. Ann Cardiothorac Surg, 2017,6(2):88-98.

[9] di Pietro M, Canto MI, Fitzgerald RC. Endoscopic Management of Early Adenocarcinoma and Squamous Cell Carcinoma of the Esophagus: Screening, Diagnosis, and Therapy[J]. Gastroenterology, 2018,154(2):421-436.

[10] Tentzeris V, Lake B, Cherian T, et al. Poor awareness of symptoms of oesophageal cancer[J]. Interact Cardiovasc Thorac Surg, 2011,12(1):32-34.

[11] Patel V, Burbridge RA. Endoscopic Approaches for Early-Stage Esophageal Cancer: Current Options[J]. Curr Oncol Rep, 2015,17(1):421. 
[12] Shimamura Y, Ikeya T, Marcon N, et al. Endoscopic diagnosis and treatment of early esophageal squamous neoplasia[J]. World J Gastrointest Endosc, 2017,9(9):438-447.

[13] Yip HC, Chiu PW. Endoscopic diagnosis and management of early squamous cell carcinoma of esophagus[J]. J Thorac Dis, 2017,9(Suppl 8):S689-S696.

[14] Shah PM, Gerdes H. Endoscopic options for early stage esophageal cancer[J]. J Gastrointest Oncol, 2015,6(1):20-30.

[15] Asada-Hirayama I, Kodashima S, Fujishiro M, et al. Narrow band imaging with magnification can pick up esophageal squamous cell carcinoma more efficiently than lugol chromoendoscopy in patients after chemoradiotherapy[J]. Diagn Ther Endosc, 2013,2013:256439.

[16] Inoue H, Kaga M, Ikeda H, et al. Magnification endoscopy in esophageal squamous cell carcinoma: a review of the intrapapillary capillary loop classification[J]. Ann Gastroenterol, 2015,28(1):41-48.

[17] Yang G, Du W, Zhang X, et al. The white substance may be a potential endoscopic marker for flat esophageal mucosal neoplastic lesions[J]. Medicine, 2018,97(35):e11885.

[18] Mikhail Davydov, Delektorskaya V, Kuvshinov YP, et al. Superficial and early cancers of the esophagus[J]. Ann N Y Acad Sci, 2014,1325:159-169.

[19] Huang FL, Yu SJ. Esophageal cancer: Risk factors, genetic association, and treatment[J]. Asian J Surg, 2018,41(3):210-215.

[20] Codipilly DC, Qin Y, Dawsey SM, et al. Screening for esophageal squamous cell carcinoma: recent advances[J]. Gastrointest Endosc, 2018,88(3):413-426.

[21] Dietrich T, Reichart PA, Scheifele C. Clinical risk factors of oral leukoplakia in a representative sample of the US population[J]. Oral Oncol, 2004,40(2):158-163.

[22] Hashibe M, Sankaranarayanan R, Thomas G, et al. Alcohol drinking, body mass index and the risk of oral leukoplakia in an Indian population[J]. Int J Cancer, 2000,88(1):129-134.

[23] Reichart PA. Identification of risk groups for oral precancer and cancer and preventive measures[J]. Clin Oral Investig, 2001,5(4):207-213.

[24] Taggart MW, Rashid A, Ross WA, et al. Oesophageal hyperkeratosis: clinicopathological associations[J]. Histopathology, 2013,63(4):463-473. 



\section{Figures}

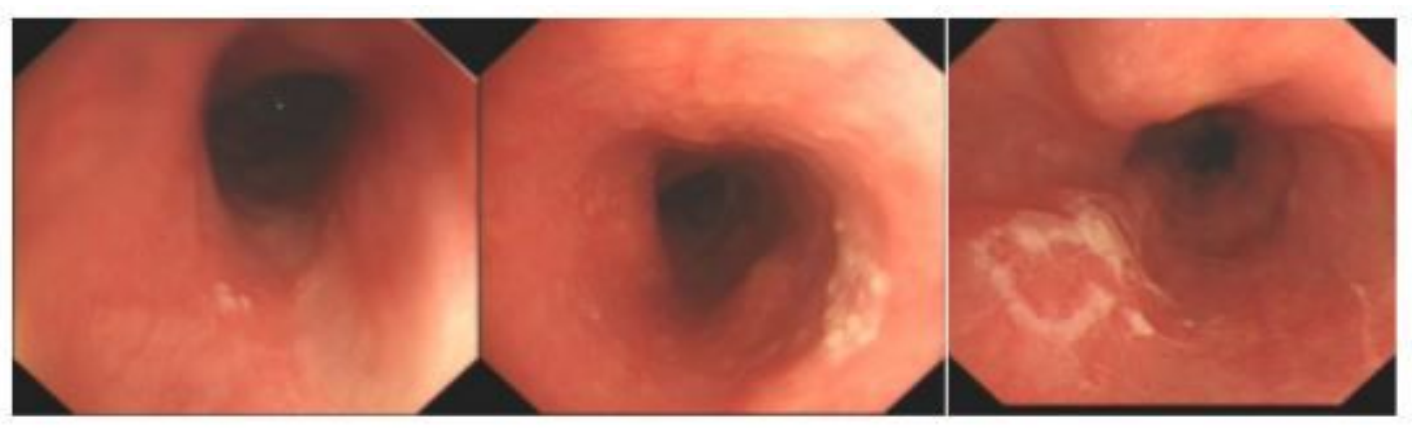

\section{Figure 1}

White substance has a clear boundary, can be distributed in flakes or as single spots, and cannot be easily washed away with water. Its shape is different from that of esophageal leukoplakia.

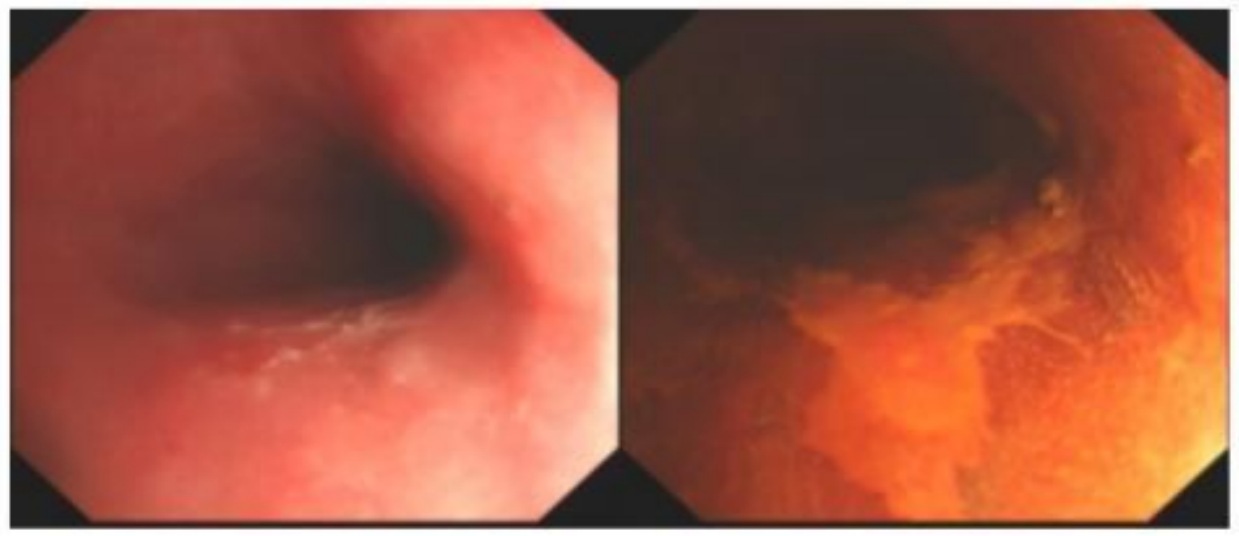

\section{Figure 2}

By white light endoscopy, the esophageal mucosa appears slightly rough and focally eroded but there is no obvious narrowing, ulcer or mass. The focal achromatic area can be detected by iodine staining.

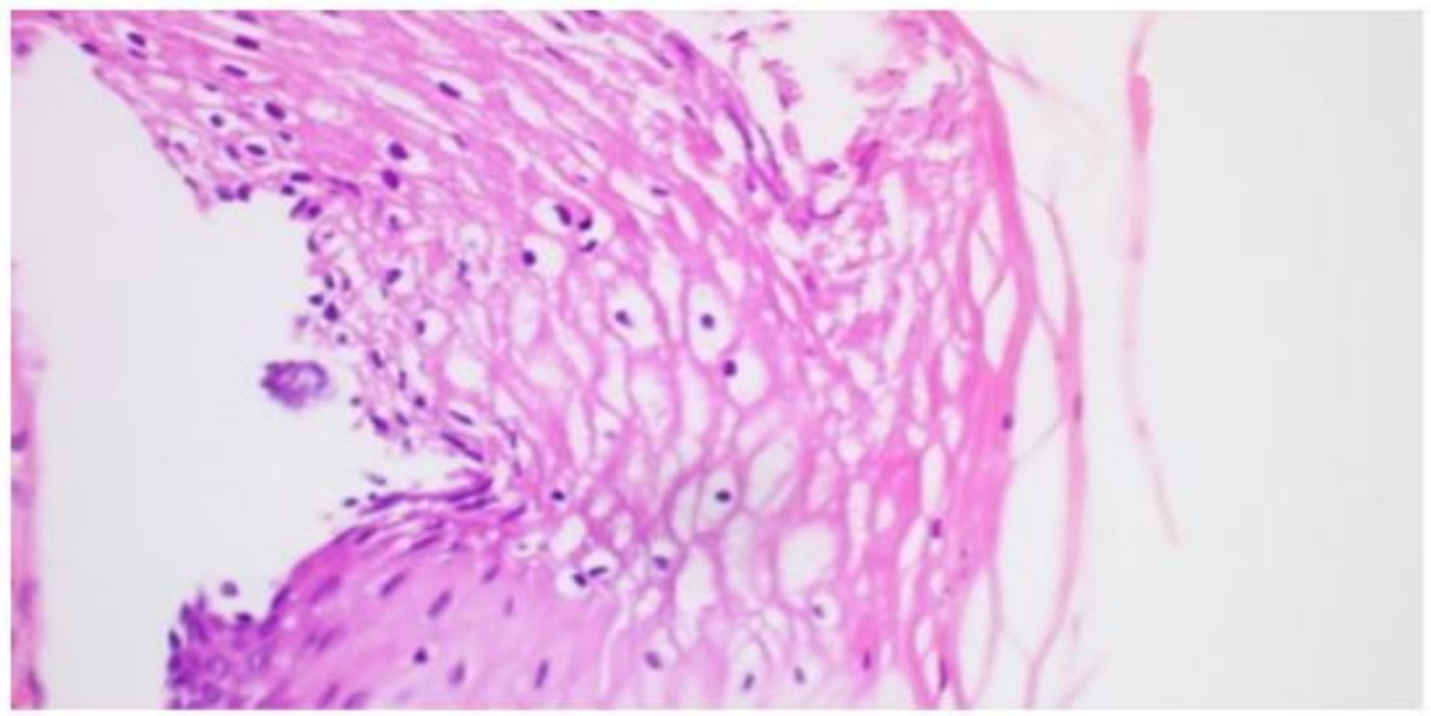




\section{Figure 3}

Hyperkeratosis (400 × magnification): the cell boundaries are unclear, the nucleus is not observable, and hyperkeratosis is clearly accompanied by the thickening of the granular layer and by obviously abnormal hyaluronic granules.

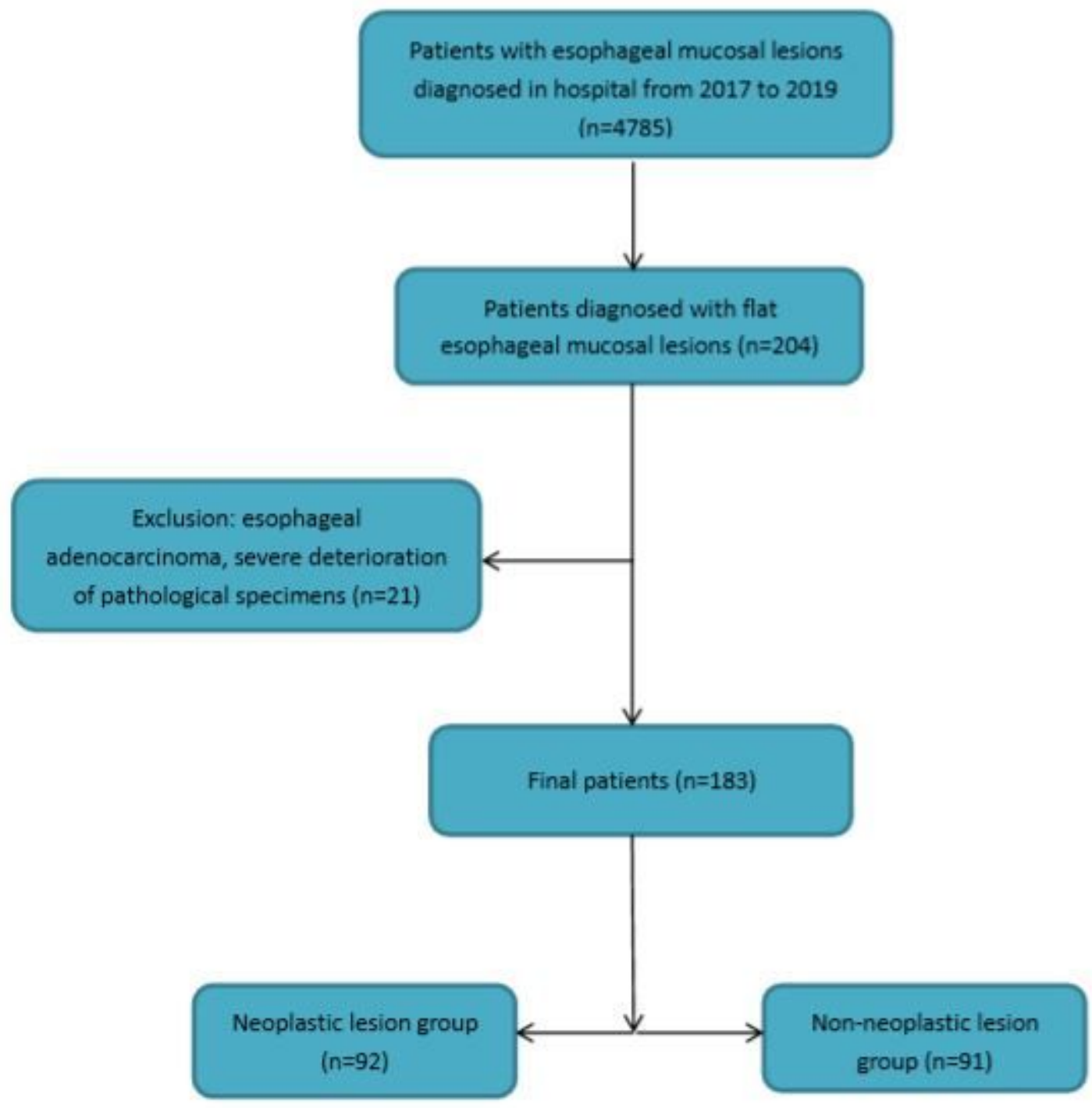

\section{Figure 4}

Flow diagram of patients enrolled in this study 


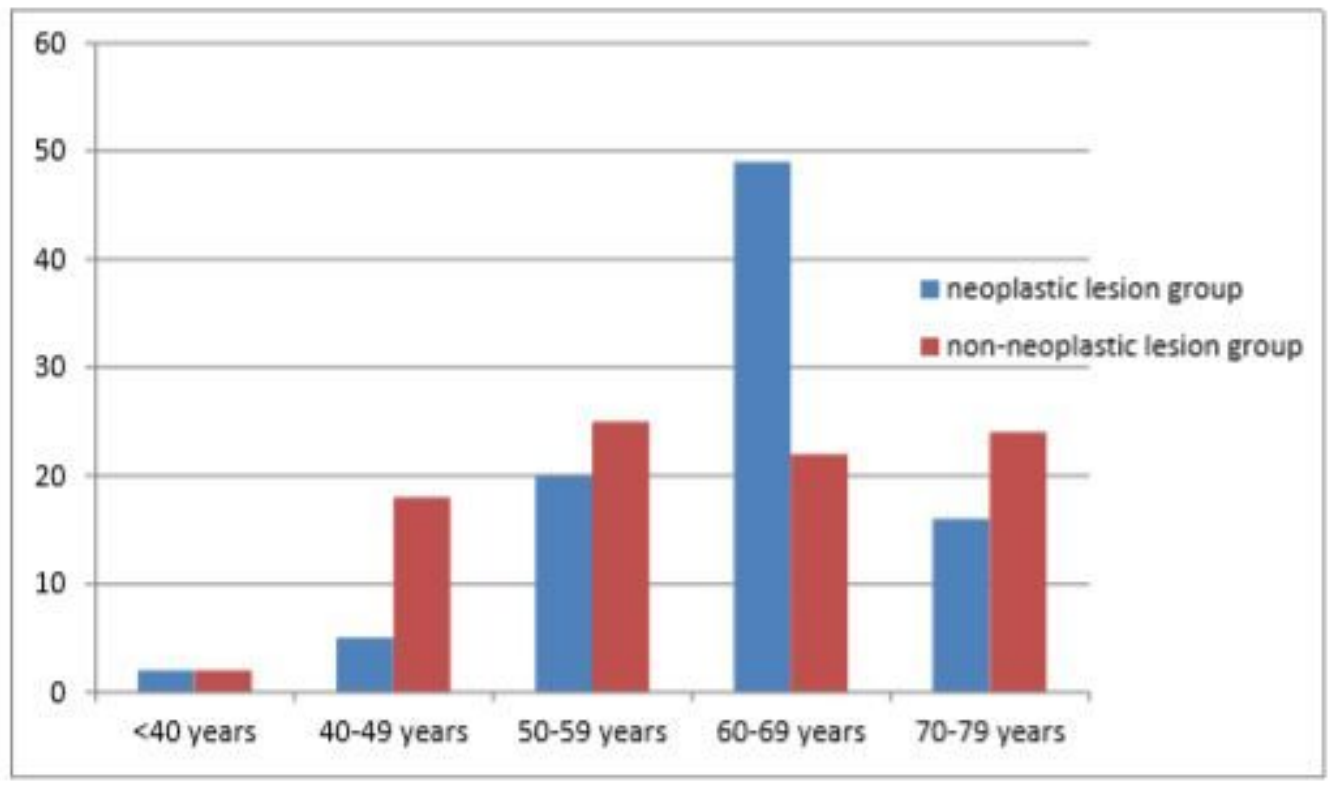

Figure 5

Age distribution of neoplastic and non-neoplastic lesions 